\title{
The Calmodulin-Stimulated Adenylate Cyclase ADCY8 Sets the Sensitivity of Zebrafish Retinal Axons to Midline Repellents and Is Required for Normal Midline Crossing
}

\author{
Hong Xu (徐洪), Sarah G. Leinwand, Alison L. Dell, Emma Fried-Cassorla, and Jonathan A. Raper \\ Department of Neuroscience, University of Pennsylvania School of Medicine, Philadelphia, Pennsylvania 19104
}

The chemokine SDF1 activates a cAMP-mediated signaling pathway that antagonizes retinal responses to the midline repellent slit. We show that knocking down the calmodulin-activated adenylate cyclase ADCY8 makes retinal axons insensitive to SDF1. Experiments in vivo using male and female zebrafish (Danio rerio) confirm a mutual antagonism between slit signaling and ADCY8-mediated signaling. Unexpectedly, knockdown of $A D C Y 8$ or another calmodulin-activated cyclase, $A D C Y 1$, induces ipsilateral misprojections of retinal axons that would normally cross the ventral midline. We demonstrate a cell-autonomous requirement for ADCY8 in retinal neurons for normal midline crossing. These findings are the first to show that ADCY8 is required for axonal pathfinding before axons reach their targets. They support a model in which ADCY8 is an essential component of a signaling pathway that opposes repellent signaling. Finally, they demonstrate that ADCY8 helps regulate retinal sensitivity to midline guidance cues.

\section{Introduction}

Axons navigate through the developing nervous system by responding to differentially localized guidance cues in their immediate environment. Many diverse families of secreted and cell surface signaling molecules have been found to guide axons through their attractive and repellent effects on growth cones (Tessier-Lavigne and Goodman, 1996; Dickson, 2002). Growth cones routinely encounter multiple cues simultaneously and must integrate their activities into unitary guidance decisions. Understanding how axons navigate through their native environment will require an understanding of how multiple cues interact with one another to affect growth cone behavior.

One such interaction is the antagonistic relationship between the chemokine SDF1 and repellent guidance cues. SDF1 has been shown to make a variety of axons less sensitive to multiple axonal repellents including semaphorin3A, semaphorin3C, and slit2 in vitro (Chalasani et al., 2003). It has also been shown to antagonize slit/robo signaling in vivo (Chalasani et al., 2007). Pharmacological studies in cultured chick sensory and retinal axons showed

Received Feb. 8, 2010; revised March 25, 2010; accepted April 3, 2010.

This work was supported by National Institutes of Health Grant 9R01-DA025407. We thank Drs. Greg Bashaw, Michael Granato, and Mathew Dalva for reading this manuscript and their comments. We thank Dr. Jin Zhang for the ICUE3 construct and Dr. Suresh Jesuthasan for the HuC:Gal4-VP16 and UAS:EGFP constructs. The Brn3c:GAP43-GFP transgenic fish line was a gift from Dr. Herwig Baier. We thank Dr. Chi-Bin Chien for the astray mutants and the Is $2 \mathrm{~b}$ :GFP transgenic fish. Radioactive in situs were performed by the Histology and Gene Expression Core of Molecular Cardiology Research Center at University of Pennsylvania with the help of Arun Padmana from Jonathan Epstein's Laboratory. We thank Drs. Sreekanth Chalasani, Mark Lush, Naomi Twery, Angela Sabol, Vanisha Lakhina, and other members of the Raper Laboratory for many thoughtful and stimulating discussions. We also thank everyone in the Granato-Mullins fish facility for their help.

Correspondence should be addressed to Jonathan A. Raper, Department of Neuroscience, University of Pennsylvania School of Medicine, 1115 BRB2/3, 421 Curie Boulevard, Philadelphia, PA 19104. E-mail: raperj@mail.med. upenn.edu.

DOI:10.1523/JNEUROSCI.0699-10.2010

Copyright $\odot 2010$ the authors $\quad 0270-6474 / 10 / 307423-11 \$ 15.00 / 0$ that the antirepellent effect of SDF1 is mediated by the seven transmembrane receptor CXCR4, a pertussis toxin-sensitive G-protein-coupled pathway, the stimulation of calcium-calmodulin, and the activation of protein kinase A (PKA). An analog of cAMP stimulates the antirepellent pathway, whereas a cAMP antagonist blocks the antirepellent effects of SDF1 (Chalasani et al., 2003). These results suggest that SDF1 signals through a calmodulin-activated intermediary to elevate cAMP. Altering levels of cyclic nucleotides has been shown to dramatically alter growth cone responses to both attractants and repellents in vitro (Song and Poo, 1999; Piper et al., 2007), but there is very little understanding of how these levels are controlled in vivo. There are 10 recognized adenylate cyclases (ADCYs) that produce cAMP in higher vertebrates (Willoughby and Cooper, 2007). Two of them, ADCY1 and ADCY8, are known to be activated when they bind calcium-activated calmodulin (Ferguson and Storm, 2004). ADCY1 is maximally activated by a combination of activated $\mathrm{G} \alpha$ s and calmodulin, whereas ADCY8 is activated by calmodulin alone (Wayman et al., 1994; Nielsen et al., 1996). Since SDF1 initiates its antirepellent effects through a pertussis toxin-sensitive $\mathrm{G} \alpha_{\mathrm{i}}$ or $\mathrm{G} \alpha_{\mathrm{o}}$, and since it is not blocked by interfering with $\mathrm{G} \alpha_{\mathrm{s}}$-mediated signaling (E. N. Twery and J. A. Raper, unpublished data), we hypothesized that the antirepellent effects of SDF1 are mediated by calmodulin-induced ADCY8 generation of cAMP. We therefore tested whether knockdown of ADCY8 interferes with SDF1-mediated antirepellent signaling in cultured zebrafish retinal axons and during retinal pathfinding in the embryonic zebrafish.

Consistent with the hypothesis that calmodulin-activated cyclases participate in the SDF1-activated antirepellent pathway, knockdown of ADCY8 makes zebrafish retinal axons more responsive to slits in the presence of SDF1 in vitro. Either ADCY8 or SDF1 knockdown tends to rescue specific retinal misprojections 
in mutant embryos in which retinal sensitivity to midline repellents is reduced. Surprisingly, knockdown of zADCY8 in the embryo induces abnormal ipsilateral retinal projections, and these misprojections can be rescued by the simultaneous reduction or elimination of slit/robo repellent signaling. These findings demonstrate a requirement for the calmodulin-activated adenylate cyclase ADCY8 in retinal axon pathfinding in vivo, and they are consistent with a model in which ADCY8 is part of a pathway that antagonizes repellent slits expressed at the ventral midline.

\section{Materials and Methods}

Fluorescence resonance energy transfer imaging. Changes in cAMP levels were monitored in retinal ganglion cells (RGCs) by fluorescence resonance energy transfer (FRET) using the cAMP sensor ICUE3 (DiPilato and Zhang, 2009). Embryonic day 5 chicken retinas were dissected and dissociated after treatment with $0.25 \%$ trypsin for $12 \mathrm{~min}$ at $37^{\circ} \mathrm{C}$. Dissociated retinal neurons were electroporated with ICUE3 plasmid using the rat neuron Nucleofector kit and plated onto the glass bottoms of MatTak dish coated with laminin. Neurons were allowed to attach after plating for $6 \mathrm{~h}$ in F12 medium supplemented with $6 \mathrm{mg} / \mathrm{ml}$ glucose, $2 \mathrm{~mm}$ glutamine, $100 \mu \mathrm{M}$ putrescine, $200 \mu \mathrm{M}$ progesterone, $5 \mu \mathrm{g} / \mathrm{ml}$ insulin, 20 $\mu \mathrm{g} / \mathrm{ml} \mathrm{NGF}, 100 \mathrm{U} / \mathrm{ml}$ penicillin, $100 \mu \mathrm{g} / \mathrm{ml}$ streptomycin, and $500 \mu \mathrm{l}$ of bovine pituitary extract (BPE) per $100 \mathrm{ml}$. The medium was then exchanged with F12 medium supplemented with all of the above components except BPE and cultured overnight at $37^{\circ} \mathrm{C}$ and $5 \% \mathrm{CO}_{2}$. On the second day, the F12 medium was replaced with L15 medium supplemented with all of the above components except BPE, and the temperature was maintained at $37^{\circ} \mathrm{C}$ on a heating stage at atmospheric $\mathrm{CO}_{2}$. Retinal ganglion cells were easily recognized as cells with long processes. Time-lapse imaging of the FRET indicator was performed on a Leica TSP2 confocal microscope. The indicator was excited at $458 \mathrm{~nm}$, and dual-emission images were collected at 465-490 nm (cyan) and 535-560 $\mathrm{nm}$ (yellow) every $30 \mathrm{~s}$. Background was subtracted from both cyan and yellow channel images, and the ratios of cyan-to-yellow emissions were then calculated for each time point and normalized to the average emission ratio just before treatment.

Zebrafish maintenance. Wild-type Tü strain zebrafish were raised and maintained according to Mullins et al. (1994). Zebrafish embryos were staged by time after fertilization and/or morphology (Kimmel et al., 1995). Heterozygous transgenic Brn3c:GAP43-GFP (Xiao et al., 2005) or Isl2b:GFP fish (Pittman et al., 2008) were used to produce embryos for cell transplantation experiments or in situ hybridization studies. Homozygous zebrafish robo2 astray ${ }^{\text {te } 284}$ or astray ${ }^{\text {ti272z }}$ mutant fish (Fricke et al., 2001) were mated to produce homozygous mutant embryos for morpholino injections. All fish lines were kindly made available by the Chien Laboratory (University of Utah Medical Center, Salt Lake City, UT). The astray mutations were identified by PCR as described previously (Chalasani et al., 2007). Fish were raised in $0.006 \%$ phenylthiourea to prevent pigmentation in embryonic and larval fish.

Cloning of zebrafish calmodulin-activated adenylate cyclases. The fulllength protein sequences and calmodulin-binding sites of human ADCY1 and ADCY8 were identified using the basic local alignment search tool (BLAST) zebrafish genomic and expressed sequence tag (EST) databases at the Sanger Centre and National Center for Biotechnology Information. Two close relatives of ADCY1 and one of ADCY8 were found. Total RNA was extracted from 48 hpf zebrafish embryos and reverse-transcribed into cDNA. Primers for reverse transcription (RT)-PCR were designed according to the zebrafish genomic and EST sequences. The sequences were completed by $5^{\prime}$ - and $3^{\prime}$-rapid amplification of cDNA ends (RACE) (BD Biosciences; K1802-1). According to the seventh version of the Ensemble zebrafish database (Zv7, release 50; http://www.ensembl.org/Danio_rerio/Info/Index), zADCY1a is located on chromosome 20, whereas both zADCY1b and zADCY8 are located on chromosome 2.

Constructs. The ICUE3 construct used as a cAMP sensor for FRET imaging was a kind gift from Dr. J. Zhang (Johns Hopkins School of Medicine, Baltimore, MD) (DiPilato and Zhang, 2009). The pHuC:Gal4VP16 and pUAS:EGFP plasmids were a kind gift from Dr. S. Jesuthasan (National University of Singapore, Singapore) (Hendricks and Je- suthasan, 2007). The pUAS:GAP43-mGFP plasmid was made to label axons by replacing the enhanced green fluorescent protein (EGFP) coding region from pUAS:EGFP with a coding sequence for GAP43-mGFP (Xiao et al., 2005). The zebrafish ADCY8 expressing plasmid pUAS: ADCY8 was made by replacing the EGFP coding region from pUAS: EGFP with the zADCY8 coding region.

Whole-mount in situ hybridization. In situ hybridization was performed as described previously (Chalasani et al., 2007) with minor modifications. Briefly, digoxigenin-labeled antisense cRNA probes were incubated with embryos to detect the expression pattern of various transcripts. Anti-DIG-POD (Roche; catalog \#11 207733 910) was applied and the signal was amplified with a cyanine 3 -coupled tyramide system method (PerkinElmer; NEL 744). Immunostaining was performed simultaneously where needed to detect the colocalization of green fluorescent protein (GFP)-labeled axons.

Immunostaining. Immunostaining was performed as previously described (Chalasani et al., 2007). Briefly, zebrafish larvae were fixed overnight in $4 \%$ paraformaldehyde (PFA) and treated with $0.2 \%$ collagenase for $2.5-3 \mathrm{~h}$ to facilitate antibody access into the tissue. Goat anti-GFP (1:500; Rockland Immunochemicals; catalog \#600-101-215) and antigoat IgG Alexa Fluor 488 (1:500; Invitrogen) were used to visualize GFPpositive cells and axons. Immunostained larvae were imaged with a Leica TSP2 confocal microscope, and all $Z$-series stacks are shown as twodimensional projections.

Antisense morpholino design, injection, and analysis. Antisense morpholinos were synthesized by Gene Tools. Two were designed to block pre-mRNA splicing and targeted exons within the first guanylate cyclase domain of zADCY8. Both cause shifts in the reading frame and almost immediate truncation of the translated protein. The morpholino sequences were as follows: E3, 5' -AAG ACA GAA ATT ACC TCA CGT TCT C-3' (underlined nucleotides correspond to exon sequence), and E4, 5'-AAG TGT GTT TAC TTA CGT GTG CCA G-3'. The sequence of the standard control morpholino from Gene Tools is $5^{\prime}$-CCT CTT ACC TCA GTT ACA ATT TAT A-3'. Morpholinos were microinjected into the yolks of one- to two-cell stage embryos immediately after fertilization at the following concentrations: E3, $1.8 \mathrm{ng} / \mathrm{embryo}$; E4, $3.2 \mathrm{ng} / \mathrm{embryo}$; and E3 plus E4, 1.0 ng E3 plus $1.5 \mathrm{ng}$ E4/embryo. To characterize the effects of morpholinos on splicing, RT-PCR was performed using cDNA templates prepared from the RNA of 48 hpf injected embryos. The PCR products were sequenced to confirm deletion of the expected exons.

Visualizing retinotectal projections. Zebrafish larvae at $5 \mathrm{dpf}$ were fixed in $4 \%$ PFA overnight and mounted in $1.3 \%$ agarose on glass slides. Retinal axons from the left eye were anterogradely labeled with the lipophilic tracer DiI (D282; Invitrogen) and those from the right eye with DiD (D7757; Invitrogen), both dissolved at $2.5 \mathrm{mg} / \mathrm{ml}$ in dimethylformamide. After injection, the dyes were allowed to diffuse overnight at room temperature. The dye-labeled retinotectal axons were scanned on a Leica TSP2 confocal microscope and all images are presented as projections of $Z$ series.

Zebrafish retinal ganglion cell culture. Zebrafish retinal explants were prepared and cultured as previously described (Chalasani et al., 2007). Control or zADCY8 morpholinos were injected into one-cell stage embryos. At $2 \mathrm{dpf}$, the embryos were anesthetized, and the eyes were removed. Retinal explants from zADCY8 and from control morpholinotreated fish larvae ( $\sim 10$ retinas per condition) were cultured on opposite halves of poly-lysine $(200 \mu \mathrm{g} / \mathrm{ml})$ - and laminin $(80 \mu \mathrm{g} / \mathrm{ml})$-coated glassbottom MatTek dishes. After $24 \mathrm{~h}$ in culture, retinal axons were allowed to grow for a $75 \mathrm{~min}$ control period and then for an additional $75 \mathrm{~min}$ treatment period after the addition of supernatants containing the following: (1) mock-transfected, (2) human slit2-transfected, (3) zebrafish SDF1a-transfected, or (4) human slit2-transfected plus zebrafish SDF1a. Images were taken with a CCD camera (Spot; Phase 3 Imaging) at the beginning of the control and treatment periods and the end of treatment period. The migration distances of retinal axons during control and experimental periods were analyzed using Image-Pro software.

Cell transplantation. Donor embryos from Brn3c:GAP43-GFP transgenic parents were injected at the one-cell stage with a mixture of rhodamine-conjugated dextran (Invitrogen; D3308) and zADCY8 morpholinos. Host embryos were uninjected wild type and stage-matched 
A

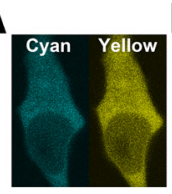

B

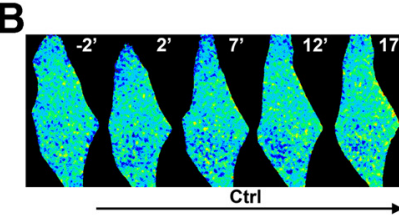

D

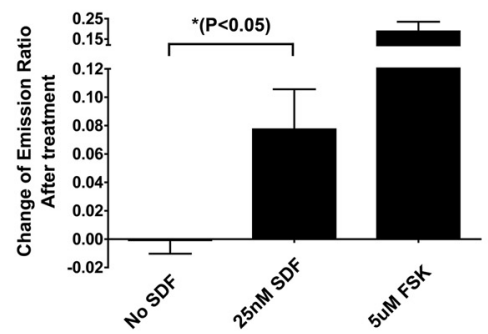

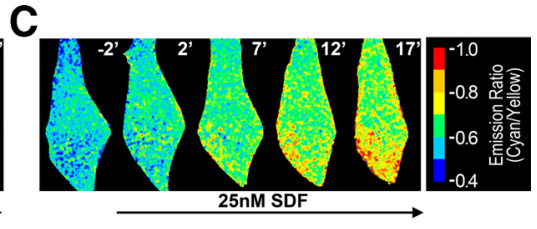

E

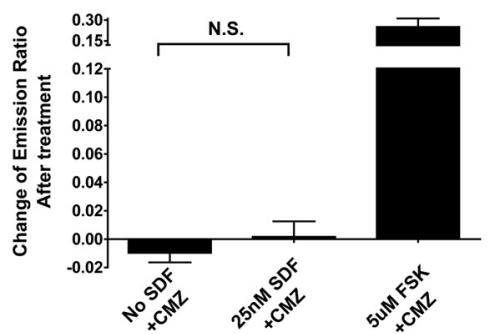

Figure 1. SDF induces a calmodulin-dependent increase in cAMP levels in retinal ganglion cells. $\boldsymbol{A}$, Example images of a chick retinal ganglion cell transfected with ICUE3, a FRET-based cAMP biosensor, in the cyan and yellow channels. B, C, Pseudocolored cyan/yellow ratio images depict FRET responses to vehicle $(\boldsymbol{B})$ or SDF $(\boldsymbol{C})$ treatment. Treatment commenced at time 0 , and images are presented at the indicated time points in minutes. $\boldsymbol{D}$, The average changes in the cyan/yellow ratio are indicated for 12 retinal ganglion cells treated first with SDF and then later with forskolin (FSK). The ratios were calculated $15 \mathrm{~min}$ after the commencement of treatment. The SEMs are indicated. There was a statistically significant difference between the change in FRET signals during the vehicle compared with the SDF1 treatment periods. The significance level was determined by the Mann-Whitney rank test. $\boldsymbol{E}$, In 12 different retinal neurons, SDF treatment induced no significant change in FRET when $8 \mu$ m calmodulin antagonist CMZ was present.

with donor embryos. At the sphere stage, embryos were dechorionated and $\sim 20-50$ donor embryo blastocysts were transplanted near the animal pole of the host, the region fated to become retina (Kozlowski et al., 1997). The donor embryos were phenotyped at $5 \mathrm{dpf}$, and the host embryos were fixed at $4 \mathrm{dpf}$ for immunostaining analysis.

Electroporation into the eye. Electroporation of the eye was performed as described with slight modifications (Hendricks and Jesuthasan, 2007). Embryos were injected at the one-cell stage with zADCY8 morpholinos. At $\sim 24 \mathrm{hpf}$, TE buffer containing the plasmids HuC:Gal4-VP16, UAS: GAP43-GFP, with or without pUAS:zADCY8 were injected into the left eye and subjected to five 13 or $14 \mathrm{~V}$ pulses $1 \mathrm{~ms}$ in duration and $500 \mathrm{~ms}$ apart using an ECM 830 square-wave electroporation system (BTX). The $\mathrm{HuC}$ promoter drives gene expression in early differentiated neurons (Park et al., 2000). At 4 dpf, the electroporated fish larvae were fixed for immunostaining and analysis.

\section{Results}

SDF1 induces a calmodulin-dependent increase in cAMP within retinal ganglion cells

Previous studies have defined the outlines of a signaling pathway through which the chemokine SDF1 reduces axonal responses to repellent guidance cues in cultured embryonic primary neurons (Chalasani et al., 2003). This pathway is unusual as it appears to require an increase in cAMP that is induced by a pertussis toxinsensitive $\mathrm{G} \alpha_{\mathrm{i} / \mathrm{o}}$-protein intermediary. This presumed increase has not been measured directly and contrasts with measured reductions of cAMP levels in SDF1-treated astrocytes and epithelial cells (Dwinell et al., 2004; Warrington et al., 2007). We used the FRET-based cAMP sensor ICUE3 (indicator of cAMP using Epac) (DiPilato and Zhang, 2009) to directly detect SDF1induced changes in cAMP levels in cultured embryonic retinal ganglion cells. An expression vector encoding ICUE3 was transfected into cultured primary chick retinal ganglion cells. The ratio of cyan to yellow fluorescence was monitored to track changes in cAMP levels. This ratio increased in SDF1-treated cells over the course of $>10 \mathrm{~min}$, indicating that cAMP levels rose in response to SDF1 treatment (Fig. $1 A-C$ ). No increase in cAMP was detected without the application of SDF1, and even greater increases were observed in response to application of the adenylate

cyclase stimulator forskolin (Fig. 1D). The SDF1-induced increase in cAMP, but not the forskolin-induced increase in cAMP, was abolished by the calmodulin antagonist calmidazolium (CMZ) (Fig. $1 E)$. These findings demonstrate that, in primary embryonic retinal ganglion cells, SDF1 can induce an increase in cAMP that is dependent on calmodulin activation. They are consistent with previous findings in embryonic neurons and support the hypothesis that the effects of SDF1 are mediated through calmodulin-activated adenylate cyclases.

\section{The identification of calmodulin-activated adenylate cyclases in zebrafish}

BLAST searches identified three ADCYs in the Ensembl zebrafish genome database that contain sequences matching calmodulin binding sites in higher vertebrate ADCY1 and ADCY8 (Vorherr et al., 1993; $\mathrm{Gu}$ and Cooper, 1999). The full coding sequences of three zebrafish ADCYs were reconstructed by $5^{\prime}$ - and $3^{\prime}$-RACE using $48 \mathrm{hpf}$ zebrafish cDNA as a template. Two of them are most closely related to human ADCY1 and the third to ADCY8, so we have named them zADCY1a, zADCY1b, and zADCY8, respectively (GenBank accession numbers: ADCY1a, GU169394; ADCY1b, GU169395; ADCY8, FJ472834). The organization of human compared with zebrafish exons and introns, guanylate cyclase domains, and calmodulin binding sites are all highly conserved (Fig. $2 A, B$; supplemental Fig. S1 $A-C$, available at www.jneurosci.org as supplemental material). The amino acid sequences of the zebrafish guanylate cyclase domains are $\sim 90 \%$ identical with those in the corresponding human ADCYs.

This study focuses on the functional characterization of zADCY8 during retinal axon outgrowth. The predicted gene structure of zADCY8 is strikingly similar to that of hADCY8. Both have 18 exons. The numbers of nucleotides in each exon are similar between fish and humans except for the first and last exons, which are both shorter in zebrafish. We have tentatively numbered the exons in zADCY8 according to their human counterparts. There are two calmodulin-binding domains, one near the $\mathrm{N}$ terminus and the other near the $\mathrm{C}$ terminus. Both are required for calmodulin regulation of enzymatic activity ( $\mathrm{Gu}$ and Cooper, 1999; Simpson et al., 2006). The N-terminal-most calmodulin-binding site is thought to recruit calmodulin to ADCY8, whereas the interaction of calmodulin with the C-terminal-most site is thought to stimulate ADCY8 cyclase activity (Simpson et al., 2006). Both calmodulin binding sites are highly conserved between zebrafish and higher vertebrates (Fig. $2 A$ ). There are only a small number of conservative substitutions that would not be expected to interfere with calmodulin binding (Rhoads and Friedberg, 1997).

\section{ADCY8 is expressed in retinal ganglion cells within the zebrafish retina}

In situs for zADCY8 in $36 \mathrm{hpf}$ zebrafish embryos demonstrate expression in the CNS as a whole and in the retinal ganglion cell layer of the eye (Fig. 2C,D). To confirm that zADCY8 is expressed in retinal ganglion cells, in situs were performed in Isl2b:GFP transgenic ze- 
brafish in which all differentiated RGCs express GFP (Pittman et al., 2008). zADCY8 transcripts were detected within individual RGC cells in 36 hpf embryos (Fig. 2E,F). This is a relatively early time when the first RGCs have been born and their axons are just beginning to cross the ventral midline (Burrill and Easter, 1995). Two non-overlapping RNA probes detected the same expression pattern for zADCY8. zADCY1b, but not zADCY1a, is also expressed at comparable levels and times in retinal ganglion cells (supplemental Fig. S1E,F, available at www.jneurosci.org as supplemental material). These results demonstrate that the calmodulin-stimulated adenylate cyclases zADCY8 and zADCY1b are expressed in RGCs at a time consistent with their playing a role in retinal axon guidance.

\section{Morpholino design for knockdown of zADCYs}

ADCYs require both guanylate cyclase domains for catalytic activity (Willoughby and Cooper, 2007). We designed morpholinos that were predicted to cause disruption of the first guanylate cyclase domain of zADCY8 by causing early exons to be skipped during the splicing of pre-mRNAs (Morcos, 2007). Skipping these exons should cause a shift in reading frame and premature termination of translation. The efficacy of each morpholino was tested by performing RT-PCR for targeted sequences using cDNA prepared from morpholino-injected compared with control 48 hpf embryos. Morpholinos targeting either exon 3 or 4 of zADCY8 were tested singly and in combination (Fig. 2B). Agarose gel electrophoresis and sequencing of the RT-PCR products indicated that each morpholino induces truncated PCR products of the expected size (Fig. $2 B$, arrowheads), loss of either exon 3 or exon 4 as expected, and generation of an early termination codon in the truncated sequences. Combining the two morpholinos induces a mixture of PCR products missing exon 3 , exon 4 , or both exons (Fig. $2 B$, arrowheads). Most subsequent knockdown experiments were performed with the two morpholinos together, but where noted, phenotypes were confirmed with single morpholinos. Comparable splice blocking morpholinos against exons 5 or 7 of zADCY1a (supplemental Fig. S1B, available at www. jneurosci.org as supplemental material) and exons 4 and 7 of zADCY1b (supplemental Fig. S1C, available at www. jneurosci.org as supplemental material) were made and tested.
A

ADCY8
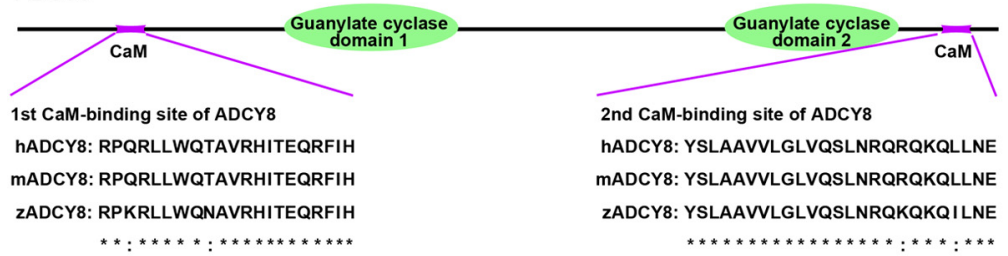

B
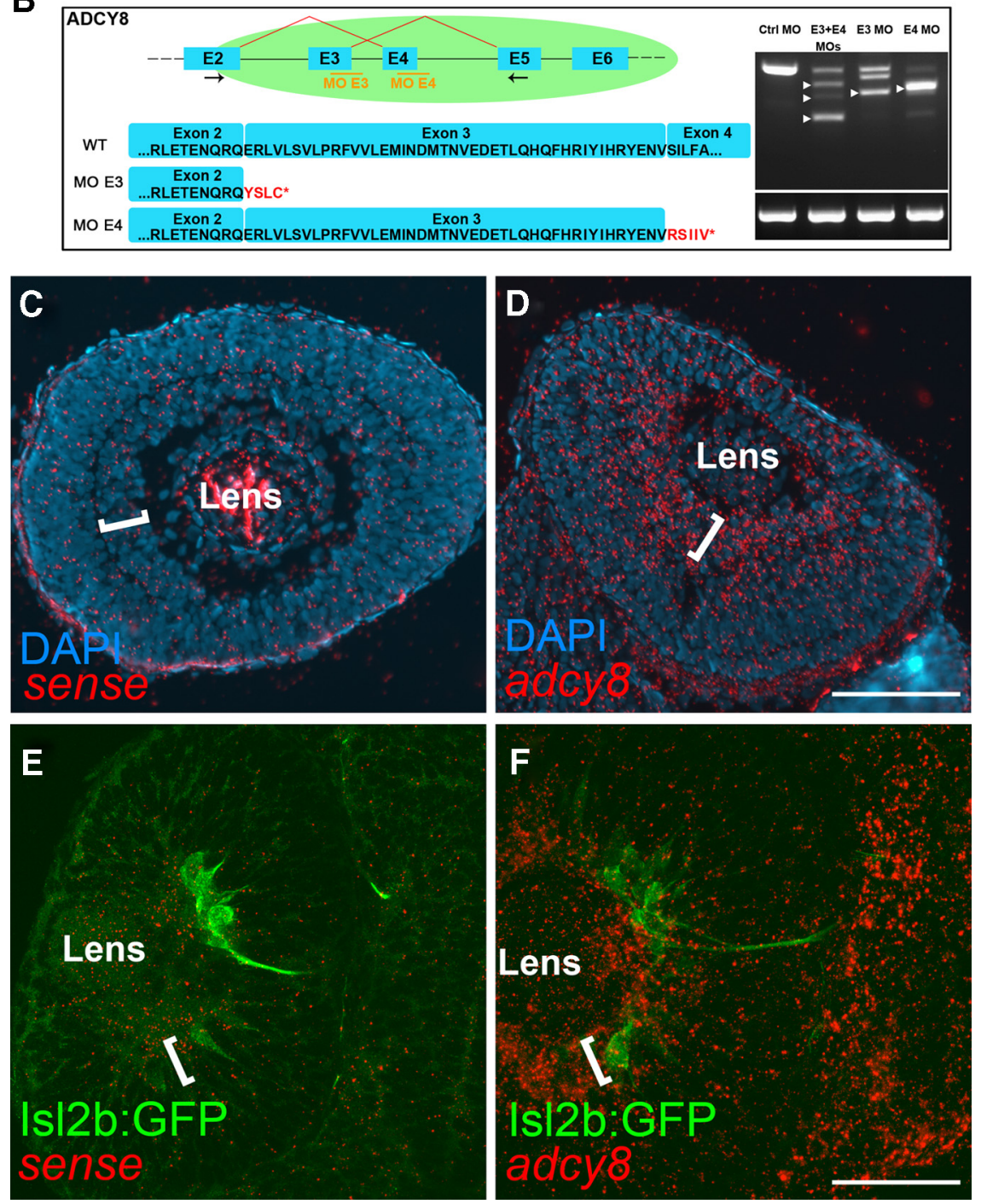

Figure 2. The identification of type 8 adenylate cyclase (ADCY8) in zebrafish and its expression in the retinal ganglion cell layer of the eye. $A$, A close relative of human ADCY 8 was identified and cloned in zebrafish. Two sequences in ZADCY8 correspond to known calmodulin-binding domains in mammalian cyclases (purple) and are highly conserved between higher vertebrates and zebrafish. $\boldsymbol{B}$, Two morpholinos were designed to block the splicing of ZADCY8 pre-mRNAs. Morpholino target sequences (orange) correspond to junctions between introns (black lines) and exons (numbered blue boxes). RT-PCR between the primers indicated with black arrows was used to detect morpholino-induced mRNA missplicing. Normal ZADCY8 sequence is represented in black lettering and truncated sequences induced by missplicing in red. Premature stop codons are indicated with asterisks. The green ovals indicate the catalytic guanylate cyclase domains in $\mathrm{ZADCY} 8$. The highest molecular weight bands generated by RT-PCR in the presence of control morpholinos arise from normally spliced transcripts, whereas lower molecular weight bands marked with arrowheads arise from morpholino-induced missplicing. PCR products generated using primers recognizing EF1 $\alpha$ in the same samples are shown below. $C-F$, zADCY8 is expressed in the retinal ganglion cell layer of zebrafish eyes at $36 \mathrm{hpf}$ when the first retinal axons cross the ventral midline. $C$, D, Paraffin-embedded $36 \mathrm{hpf}$ embryos were sectioned and stained with DAPI $\left(4^{\prime}, 6^{\prime}-\right.$ diamidino-2-phenylindole) to visualize nuclei. The binding of radiolabeled sense or antisense zADCY8 probes were visualized in a photoemulsion overlay by dark-field microscopy. Specific ZADCY8 mRNA expression was detected in the retinal ganglion cell layer (brackets) in the eye. Variable nonspecific binding of both sense and antisense probes was noted in the lens. $\boldsymbol{E}, \boldsymbol{F}$, Whole-mount Is|2b:GFP transgenic fish in which retinal ganglion cells are labeled (green) were probed with sense and antisense ZADCY 8 probes (red). Fluorescent images representing projected Z-stacks $5 \mu \mathrm{m}$ deep demonstrate that ZADCY8 mRNA is detected within individual retinal ganglion cells ( $\boldsymbol{F}$, brackets) viewed from the ventral side of the embryo with anterior to the top. Scale bars, $50 \mu \mathrm{m}$. 


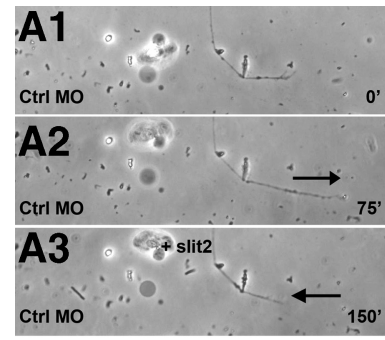

D CtrIMO CtrIMO ADCY8MO ADCY8MO + +slit2 $\quad+$ slit2
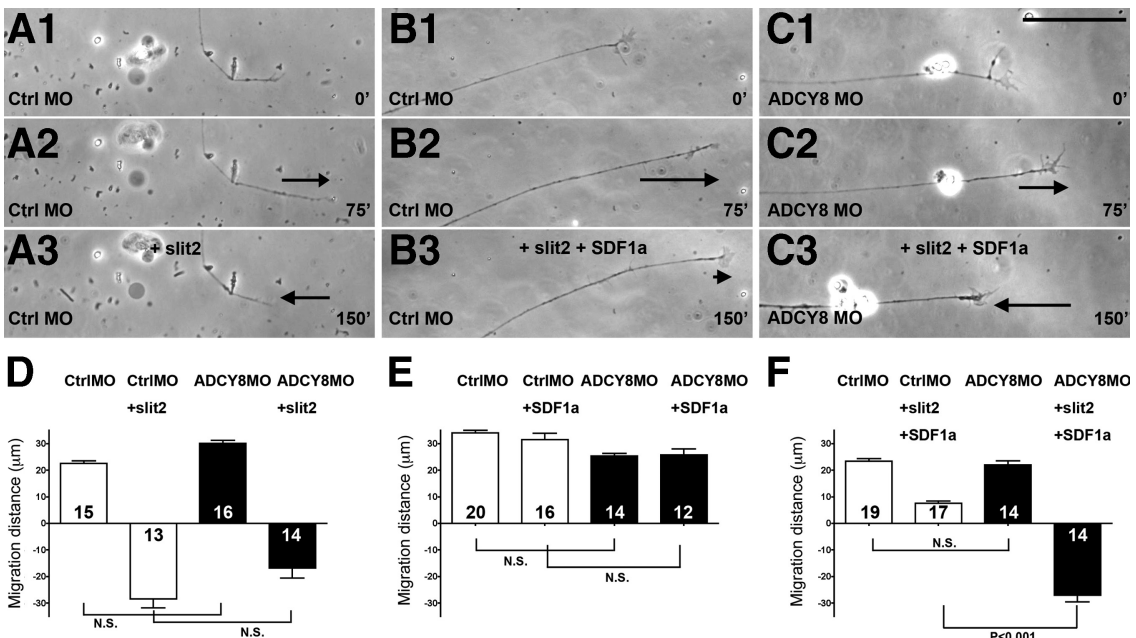

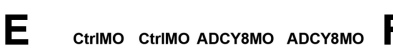

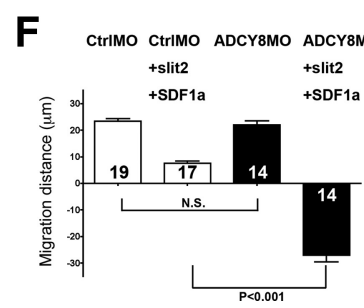

Figure 3. Knockdown of ZADCY8 blocks the antirepellent effect of SDF1a in vitro. Retinal explants were prepared from $50 \mathrm{hpf}$ control morpholino or ZADCY 8 morpholino-injected embryos and cultured for $24 \mathrm{~h}$ in vitro. Cultures were photographed at the beginning and end of a $75 \mathrm{~min}$ control period, treated over the course of an additional 75 min period with either $h S l i t 2, z S D F 1 a$, or both hSlit2 and zSDF1a, and photographed a final time. A1-A3, A control morpholino-treated retinal axon advances during the control period $(\boldsymbol{A 1}, \boldsymbol{A} \mathbf{2})$ and retracts in response to hSlit2 during the treatment period $(\boldsymbol{A} \mathbf{2}, \boldsymbol{A 3})$. $\boldsymbol{B} \mathbf{1}-\mathbf{B} 3, A$ control morpholinotreated axon fails to respond to $\mathrm{hSlit2} 2$ in the presence of zSDF1a. C1-C3, A ZADCY8 morpholino-treated axon responds to hSlit2 in the presence of zSDF1a. $\boldsymbol{D}-\boldsymbol{F}$, Quantitative comparisons of growth cone advance in the presence or absence of hSlit2 and/or zSDF1a. D, Retinal axons containing the control morpholino (open bars) or the zADCY8 morpholino (filled bars) retract equally well in response to $\mathrm{hSlit2}$. $\boldsymbol{E}$, Retinal axons containing the control morpholino or the zADCY8 morpholino do not change their rate of outgrowth in response to zSDF1a. $\boldsymbol{F}$, Retinal axons containing the control morpholino do not retract in response to a combination of hSlit2 and zSDF1a, whereas axons containing the ZADCY8 morpholino do retract, showing that retinal axons containing the ZADCY8 morpholino are more sensitive to slit2 in the presence of SDF1 than axons containing control morpholino. The number of axons analyzed for each condition is indicated within each bar. The SEM for each condition is indicated by a bar. All experiments were repeated at least three times. Pairwise levels of significance were estimated using the Mann-Whitney $U$ test. N.S., Not significantly different. Scale bar, $50 \mu \mathrm{m}$

\section{Knockdown of zADCY8 attenuates the antirepellent effect of SDF1a in vitro}

We hypothesized that SDF1-induced calmodulin activation stimulates ZADCY8 and that the resulting elevation of cAMP causes retinal axons to be relatively insensitive to repellent signals. We therefore tested whether zADCY8 knockdown makes zebrafish retinal ganglion cell axons less responsive to the antirepellent effects of SDF1a. Retinas were harvested from zADCY8 morpholino- or control morpholino-injected larvae at $2 \mathrm{dpf}$, cut into small pieces, and cultured on laminin-coated glass. Each coverslip had control explants on one side and zADCY8 morpholino-containing explants on the other (see Materials and Methods). Many individual growth cones were photographed at the beginning and end of a 75 min nontreatment period, the bathing medium was changed, and the same growth cones were photographed again after a second 75 min treatment period (Fig. $3 A 1-C 3)$. These photographs were used to measure the forward progress of individual growth cones during the two periods. During the treatment period, either the repellent slit2, the chemokine SDF1a, or both were added to the cultures.

Knockdown of ZADCY8 does not affect the rate of retinal axon outgrowth (Fig. 3D-F, compare the average migration distances between control or zADCY8 morpholino-treated axons during the nontreatment periods). Retinal axons containing control or zADCY8 morpholino respond equally well to the repellent slit2 (Fig. 3D). Retinal axons containing control or zADCY8 morpholinos advance at the same rate in the presence of zSDF1a (Fig. 3E). Consistent with our previous findings (Chalasani et al., 2007) retinal axons containing control morpholino are significantly less responsive to slit2 in the presence of $\mathrm{zSDF} 1 \mathrm{a}$ compared with its absence (Fig. 3D,F, compare empty columns). In contrast, retinal axons containing zADCY8 morpholino are equally sensitive to slit 2 in the presence of zSDF1a compared with its absence (Fig. 3D,F, compare filled columns). The difference is most apparent in experiments in which both slit2 and zSDF1 are presented together during the experimental period (Fig. 3F). Control morpholino-containing retinal axons continue to advance, whereas zADCY8 morpholino-containing axons retract. These findings demonstrate that ZADCY8 knockdown makes zebrafish retinal ganglion cell axons insensitive to SDF1a. They are consistent with zADCY8 serving as an essential step in the SDF1a-mediated antirepellent pathway. Importantly, they demonstrate a cell-autonomous change in retinal ganglion cell responsiveness to a repellent guidance cue when zADCY8 is knocked down.

\section{Knockdown of zADCY8 induces abnormal ipsilateral retinal axon projections}

We examined the consequences of zADCY8 knockdown on axonal pathfinding in the zebrafish retinal projection. After exiting the eye, retinal axons extend through the ventral diencephalon toward the ventral surface of the brain where they cross the midline at the optic chiasm. As they continue to extend, they grow dorsally and posteriorly to the tectum. In contrast to binocular animals, all retinal axons cross the midline in the zebrafish. We used anterograde dye tracing in $5 \mathrm{dpf}$ zebrafish larvae to examine retinal axon trajectories in zADCY8 morphants. All retinal axons crossed the midline normally and projected to the contralateral tectum in control morpholino-treated larvae (Fig. $4 A$ ). However, in a significant number of zADCY8 morphants, some or all retinal axons failed to cross the midline and misprojected instead to the ipsilateral tectum (Fig. $4 B-D$ ). Abnormal ipsilateral projections were detected in $62 \%$ of E3 morpholinotreated eyes and in 12\% of E4 morpholino-treated eyes (Fig. $4 E$ ). Combining the $\mathrm{E} 3$ and $\mathrm{E} 4$ morpholinos, using reduced amounts of each that individually produced no ipsilateral misprojections, produced robust ipsilateral misprojections. Synergy between the E3 and E4 morpholinos is consistent with their inducing their effects by acting on a common target. This combination of morpholinos is less likely to induce off-target effects than higher concentrations of single morpholinos and was therefore used in most subsequent experiments. Although misprojecting retinal axons did not cross the midline, they joined the normal ipsilateral optic tract and projected with grossly normal retinotopy into the ipsilateral tectum (supplemental Fig. S2, available at www.jneurosci.org as supplemental material).

Knocking down ZADCY8 caused a marked reduction in the overall size of the embryo (supplemental Fig. S4E, available at www.jneurosci.org as supplemental material). This effect could in principle be caused by the knockdown of zADCY8 itself since it is widely expressed, especially in the CNS, or by a toxic reaction to morpholinos that induces p53-mediated apoptotic cell death 

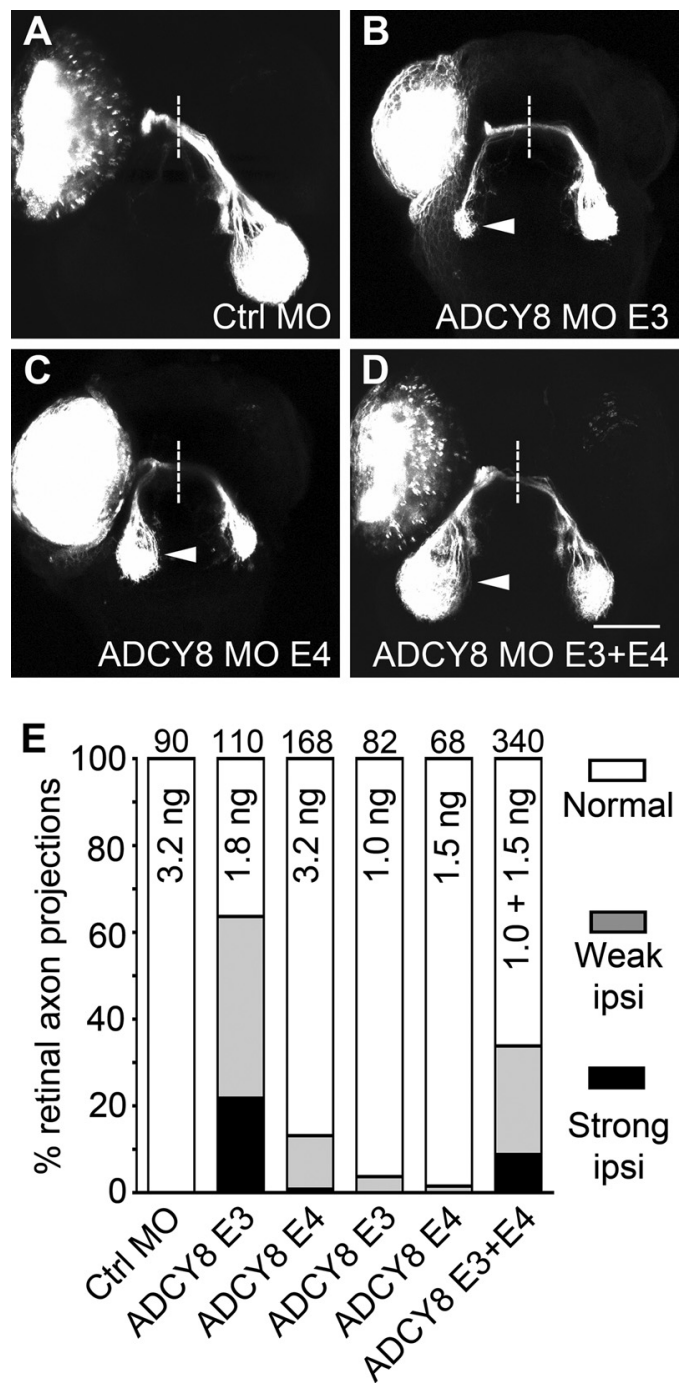

Figure 4. Knockdown of ZADCY8 induces ipsilateral misprojections of retinal axons. A-D, Representative examples of retinal axon projections in control and zADCY 8 morpholino-treated zebrafish larvae. Embryos at the one- to two-cell stage were microinjected with the indicated morpholino, fixed at $5 \mathrm{dpf}$, and retinal axons were labeled with the lipophilic dyes Dil (red) and DiD (blue). All images are dorsal views with anterior to the top. $A$, In control morpholino-treated larvae, all axons cross the midline and project contralaterally to the opposite tectum. $\boldsymbol{B}$, In a larvae treated with the $\mathrm{E} 3 \mathrm{ZADCY} 8$ morpholino, some retinal axons fail to cross the midline and instead misproject to ipsilateral tecta. The arrowheads indicate ipsilateral misprojections. C, D, Treatment with the E4 ZADCY8 morpholino produces similar ipsilateral misprojections $(\boldsymbol{C})$, as does combining low doses of the $\mathrm{E} 3$ and $E 4$ ZADCY 8 morpholinos that by themselves induce few pathfinding errors (D).E, The proportions of normal, weak ipsilateral $(<50 \%$ ipsilateral retinal axons), and strong ipsilateral ( $>50 \%$ ipsilateral axons) projections induced by ZADCY 8 knockdown are indicated. The amount of each morpholino used per embryo is indicated within each column, whereas the number of eyes examined in each condition is indicated above each column. Scale bar, $100 \mu \mathrm{m}$.

(Robu et al., 2007). We coinjected a p53 morpholino and zADCY8 morpholinos together to reduce potential p53-mediated apoptotic cell death (Robu et al., 2007) and found that the eyes and head were still small in morphant compared with wild-type embryos. Ipsilateral retinal misprojections were observed at comparable frequencies in embryos containing zADCY8 or concurrent zADCY8 and p53 morpholinos (data not shown). Knockdown of zADCY8 also induces a developmental delay in the eye. The initial formation of the optic chiasm, which occurs $\sim 36 \mathrm{hpf}$ in normal embryos, did not occur until $18 \mathrm{~h}$ later in zADCY8 morphants. The delay appeared to be greater in the eye compared with the trunk as judged by the expression pattern of cxcr4b in the lateral line primordium (David et al., 2002) (data not shown). These results suggest that zADCY8 knockdown affects both the size and rate of development of the eye and other central nervous tissues. Subsequent experiments were therefore aimed at determining whether the retinal axon misguidance phenotype is induced directly by the loss of zADCY8 in retinal ganglion cells or as an indirect consequence of developmental perturbations along the retinal pathway.

\section{Potential axon guidance cues and receptors are expressed normally in zADCY8 morphants}

Knockdown of zADCY8 might alter retinal responses to midline guidance cues or, alternatively, alter the distributions of those cues at the midline. Since there is a significant developmental delay in zADCY8 morphants, we precisely stage-matched zADCY8 morphants with wild-type embryos according to key morphological markers (Kimmel et al., 1995) and the maturity of retinal axon projections as visualized in Isl2b:GFP transgenics (Pittman et al., 2008). We compared a number of midline markers and/or potential guidance cues in normal and morphant larvae at the time when the first retinal axons cross the midline equivalent to $36 \mathrm{hpf}$ in untreated embryos. These included the midline morphogen and potential guidance cues sonic hedgehog (Trousse et al., 2001), nkx2.2 (Marcus et al., 1999), sema3d (Sakai and Halloran, 2006), and the likely repellent cues slit1a, slit1b, slit2, and slit3 (Hutson and Chien, 2002; Hutson et al., 2003). We found that each of these signaling molecules is expressed at the midline in similar patterns in both wild-type and zADCY8 morphant embryos (supplemental Fig. $\mathrm{S} 3 A-C^{\prime}, G-J^{\prime}$, available at www.jneurosci.org as supplemental material). We also examined the expression patterns of two receptors expressed in retinal ganglion cells, robo 2 and cxcr4b, each of which is required for normal retinal axon guidance (Fricke et al., 2001; Li et al., 2005). Both were detected in the RGC layer and their expression patterns are comparable between wild-type and zADCY8 morphants (supplemental Fig. S3E-F', available at www.jneurosci.org as supplemental material). Another potential retinal axon guidance receptor, neuropilin1a (Liu et al., 2004), also maintains a normal expression pattern in zADCY8 morphants (supplemental Fig. $\mathrm{S} 3 D, D^{\prime}$, available at www.jneurosci.org as supplemental material). All of these key cues and receptors are expressed in their appropriate patterns at the actual time that retinal axons first cross the ventral midline. What is more, their normal expression suggests that midline tissues differentiate normally. The errors we see in retinal pathfinding are therefore difficult to explain by the disruption of midline tissues or from the loss of known midline guidance cues or their receptors.

\section{Transplantation studies support a cell-autonomous} requirement for $\mathrm{zADCY} 8$ in retinal ganglion cells

We took two independent approaches to determine whether knockdown of zADCY8 alters retinal axon responses to midline guidance cues. In the first, we tested whether retinal ganglion cells harvested from zADCY8 morphant embryos and transplanted into untreated wild-type embryos extend axons normally across the ventral midline or whether they misproject ipsilaterally. Multipotent progenitors were harvested from Brn3c:GAP43-GFP transgenic fish in which a fluorescent axonal tracer is expressed in $\sim 50 \%$ of cells that differentiate into retinal ganglion cells (Xiao et al., 2005). These donor embryos were injected at the one-cell stage with zADCY8 morpholinos and dextran red. Blastocyst cells were harvested at $4 \mathrm{hpf}$ and transplanted into stage-matched 

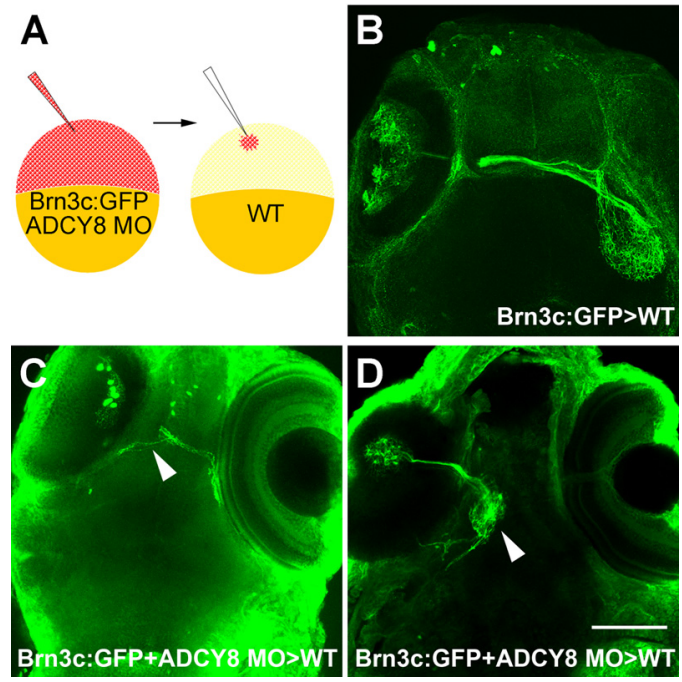

Figure 5. Retinal neurons transplanted from morphant embryos into untreated embryos generate ipsilateral misprojections. $A$, Just fertilized transgenic Brn3c:GFP eggs were injected with dextran red alone or with dextran red and zADCY8 morpholino. Cells were harvested at 4 hpf and transplanted into stage-matched untreated wild-type host embryos. $\boldsymbol{B}$, Transgenelabeled transplanted retinal axons containing no morpholino always project contralaterally at 4 dpf. $\boldsymbol{C}, \boldsymbol{D}$, Transplanted retinal axons containing zADCY8 morpholino were observed to misproject ipsilaterally in $\sim 18 \%$ of embryos examined (arrowheads). Scale bar, $100 \mu \mathrm{m}$.

wild-type host embryos near the animal pole (Fig. 5A). Chimeric embryos were allowed to grow to 4 dpf. Embryos with GFPlabeled retinal ganglion cells in only one eye were selected using a dissecting fluorescent microscope for analysis. GFP staining was enhanced by immunofluorescence and examined with a confocal fluorescent microscope. Transplanted zADCY8 morpholinocontaining retinal ganglion cell axons with ipsilateral misprojections were detected in 7 of 39 embryos (Fig. $5 C, D$ ). Since only a very small proportion of retinal neurons derive from the transplant, and since most axons project normally even when all retinal neurons contain zADCY8 morpholino, this approach would be expected to underestimate the rate at which misprojections occur when morpholino-containing retinal axons extend in normal tissues. Transplanted retinal axon trajectories that did not contain zADCY8 morpholino projected normally across the midline in 32 of 32 embryos (Fig. $5 B$ ). These results are significant to the $p<0.05$ level (Fisher's exact test) and are consistent with a retinal ganglion cell-autonomous requirement for $\mathrm{ZADCY} 8$ for normal midline crossing. Furthermore, they strongly suggest that ipsilateral misprojections in morpholino-treated embryos do not arise from a general delay in embryonic development, since ipsilateral projections occurred in chimeric embryos in which the overall rate of development was normal. However, since a small number of scattered dextran-labeled transplanted cells were generally present near the ventral midline in chimeric embryos, these results do not absolutely rule out the possibility that morpholinotreated midline cells contribute to the formation of ipsilateral misprojections. We were unsuccessful in attempts to transplant wild-type blastocyst cells into zADCY8 morpholino-treated embryos, as the recipient embryos were too fragile to survive the transplantation procedure.

Localized expression of full-length zADCY8 within the eye rescues ipsilateral misprojections of retinal axons in ZADCY8 morphants

We used a second approach to confirm that zADCY8 is required within retinal ganglion cells for normal pathfinding. Full-length
A
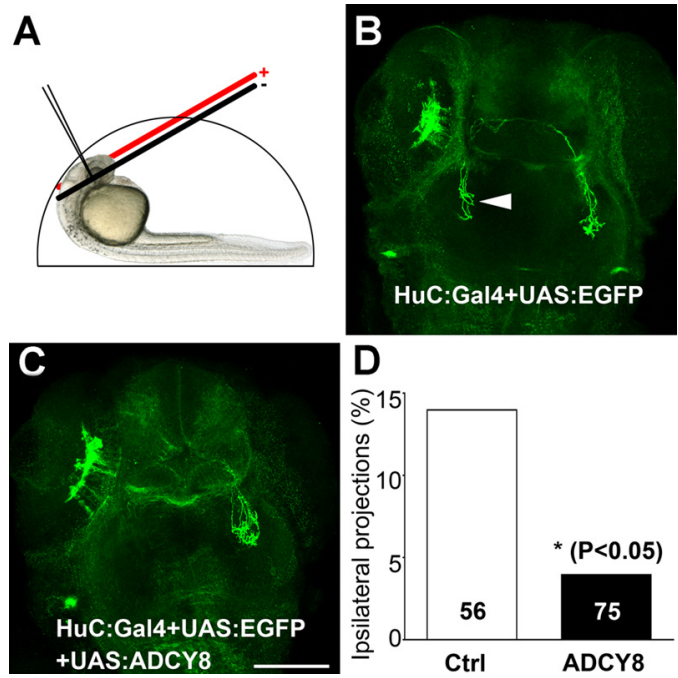

Figure 6. Expression of full-length $z A D C Y 8$ in retinal neurons corrects ipsilateral misprojections in zADCY8 morphants. A, Either HuC:Gal4+UAS:GAP43-GFP or HuC:Gal4+UAS:GAP43-GFP+UAS: $A D C Y 8$ were electroporated into one eye of ZADCY8 morphant embryos. Embryos were analyzed at 4 dpf by immunostaining for GFP. $\boldsymbol{B}$, Retinal axons expressing only GFP sometimes misproject ipsilaterally in $A D C Y 8$ morphants. $C$, ZADCY8 morphants in which retinal axons express GFP and full-length $\mathrm{ZADCY} 8$ rarely misproject ipsilaterally. $D, \mathrm{ZADCY} 8$ expression within the eye significantly reduced the frequency of ipsilateral retinal misprojections in zADCY8 morphants ( $p<0.05$, Fisher's exact test). The numbers in the bars indicate the number of morphant embryos analyzed. The control and ADCY8 plasmids are expressed in similar numbers of retinal ganglion cells in the eye. Scale bar, $100 \mu \mathrm{m}$.

zADCY8 was transfected into retinal neurons of morpholinotreated embryos to determine whether its expression rescues midline guidance errors. zADCY8 morpholino-treated $24 \mathrm{hpf}$ embryos were injected in one eye with a combination of expression plasmids containing either HuC:Gal4-VP16 and UAS:GAP43-GFP; or with HuC: Gal4-VP16, UAS:GAP43-GFP, and UAS:zADCY8 plasmids. The $\mathrm{HuC}$ promoter drives expression in neurons just before they extend axonal processes (Park et al., 2000). The injected eye was immediately electroporated to transfect the DNA into retinal neurons (Fig. $6 A$ ). Expression of electroporated constructs was detected in only a small number of retinal ganglion cells. In one experiment in which we quantified expressing cells, only an average of 2.2 ganglion cells expressed the control GFP construct and 2.2 cells expressed ADCY8. Ipsilaterally misprojecting retinal axons were detected in 14\% (8 of 56) of zADCY8 morphant embryos expressing only the control GFP construct (Fig. 6B,D). This low frequency of ipsilateral misprojections can be explained by the low proportion of retinal neurons that were labeled, since most retinal axons project contralaterally even in morphant embryos. Ipsilaterally misprojecting axons were detected in only 4\% (3 of 75) of morphant embryos in which full-length zADCY8 was transfected into the retina (Fig. 6C,D). Expression of full-length zADCY8 in nonmorphant embryos did not affect retinal midline crossing. Thus, expression of full-length zADCY8 rescued ipsilateral misprojections in morphant embryos. These results argue strongly that ipsilateral misprojections are not caused by a general delay of development in morphant embryos since rescue was successfully accomplished without reversing the overall developmental delay. They support the idea that zADCY8 activity within retinal ganglion cells influences the behavior of their axons at the midline.

\section{zADCY8 works together with $\mathrm{zADCY} 1 \mathrm{~b}$ to facilitate retinal midline crossing}

We tested whether the calmodulin-activated cyclases zADCY1a or ZADCY1b contribute to normal guidance of retinal axons at the midline. Multiple splice-altering morpholinos were con- 
firmed to induce premature stop codons within the first of their two cyclase domains (supplemental Fig. $\mathrm{S} 1 B, C$, available at www.jneurosci.org as supplemental material). As expected from the absence of zADCY1a expression in retinal ganglion cells, zADCY1a morpholinos, even at excessive concentrations that caused $\sim 50 \%$ mortality and induced reduced eye and brain size in the surviving embryos (supplemental Fig. S4C, available at www.jneurosci.org as supplemental material), produced almost no ipsilateral misprojecting retinal axons (supplemental Fig. S4 $H, K$, available at www.jneurosci.org as supplemental material). The same was true for a morpholino targeting sema3al (supplemental Fig. S4 $B, G, K$, available at www. jneurosci.org as supplemental material). In contrast, morpholinos directed against zADCY1b did induce ipsilateral retinal misprojections in $22 \%$ of morphants (supplemental Fig. S4 $I, K$, available at www. jneurosci.org as supplemental material). Since zADCY1b and zADCY8 morphants phenocopied one another, we next tested whether these two cyclases act cooperatively to promote midline crossing. Combining morpholinos to zADCY1b and $\mathrm{ZADCY} 8$ at doses too low to induce ipsilateral misprojections on their own, induced ipsilateral misprojections in $28 \%$ of morphants. These findings suggest that the calmodulin-activated cyclases zADCY8 and zADCY1b work together to promote retinal axon crossing of the ventral midline.

\section{Knockdown of zADCY8 rescues retinal projection errors in} astray mutants with partial loss of robo2 function

Slit is a repellent for retinal ganglion cell axons whose expression near the ventral midline helps determine where the optic chiasm forms (Erskine et al., 2000; Niclou et al., 2000; Hutson and Chien, 2002; Plump et al., 2002; Chalasani et al., 2003, 2007). Robo2 is the only slit receptor expressed in retinal ganglion cells as their axons cross the ventral midline in zebrafish (Lee et al., 2001). Zebrafish that have a partial loss of robo2 function display a number of retinal guidance errors, some of which can be rescued by interrupting SDF1a signaling (Chalasani et al., 2007). If zADCY8 is an essential component within the SDF1 signaling pathway, we predicted that its knockdown should tend to rescue the partial loss of robo2 phenotype. There are several retinal axon pathfinding errors in partial loss of robo2 astray ${ }^{\text {te284 }}$ mutants, including extra midline crossings, anterior and posterior misprojections, and some ipsilateral misprojections (Fig. 7A) (Fricke et al., 2001). Knocking down SDF1a or its receptor CXCR4b partially rescues the anterior misprojections in astray ${ }^{\text {te } 284}$ mutants (Chalasani et al., 2007). We focused on this specific class of pathfinding errors when we assessed the effect of ZADCY8 knockdown on astray fish. We found that the injection of zADCY8 morpholinos into astray $^{\text {te } 284}$ mutant embryos induces significant rescue of anterior misprojections (Fig. 7B,C).

Knockdown of zADCY8 does not rescue retinal projection errors in astray mutants with full loss of robo2 function Neither SDF1a or CXCR4b knockdown rescues anterior misprojections of retinal axons in zebrafish that lack functional robo2 altogether, presumably because there is no residual slit/ robo signaling that can be strengthened by reducing SDF1 signaling (Chalasani et al., 2007). If zADCY8 is a key step in the SDF1 signaling pathway, then zADCY8 knockdown would not be expected to rescue anterior misprojections in mutants with a complete loss of robo2. In robo2-null mutants, such as astray $^{\text {ti272z }}$, retinal axon pathfinding errors are more severe than in astray ${ }^{\text {te284 }}$ mutant embryos (Fig. 7D) (Fricke et al., 2001). zADCY8 knockdown has no significant effect on anterior misprojections of retinal axons in astray ${ }^{\text {ti272z }}$ mutant embryos (Fig. 7E,F). RT-PCR was performed to confirm that morpholinos knocked down zADCY8 in both astray ${ }^{\text {te284 }}$ and astray ${ }^{\text {ti272z }}$ mutants (data not shown).

\section{Knockdown of zADCY8 induces fewer ipsilateral retinal misprojections in mutants with either partial or complete loss of robo2 function}

Since ADCY8 is part of a signaling pathway that reduces axonal responses to repellents, its knockdown would be expected to make retinal axons more sensitive to repellent cues. Ipsilateral misprojections in zADCY8 morphant embryos could potentially be explained by retinal axon hypersensitivity to repellents at the midline. Slits are potent repellents that are expressed at the midline. We therefore tested whether reducing or eliminating slit signaling in robo 2 mutants reduces ipsilateral misprojections in zADCY8 morphants. We limited our analysis to strong ipsilateral misprojections in morphant embryos, defined as $>50 \%$ of retinal axons projecting ipsilaterally, since smaller ipsilateral misprojections sometimes occur in robo 2 mutants. Morpholino-induced strong ipsilateral misprojections are less prevalent in either astray $^{\text {te } 284}$ mutants that have a partial loss of robo 2 or in astray $^{\text {ti272z }}$ mutants that have a complete loss of robo2 compared with wild-type embryos (Fig. $8 D$ ). These observations are consistent with the idea that $\mathrm{ZADCY} 8$ is part of a signaling pathway that antagonizes slit/robo-mediated repellent signaling. 

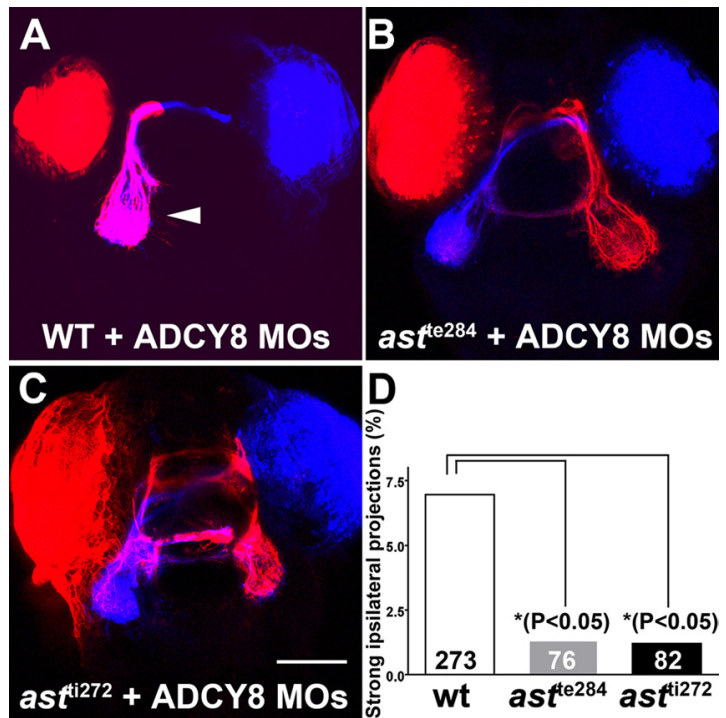

Figure 8. Ipsilateral misprojections induced by $Z A D C Y 8$ knockdown are rescued in astray mutants that have either a partial or total loss of robo2 function. $A$, An example of a strong ipsilateral misprojection (arrowhead) induced by zADCY8 knockdown in a wild-type larvae. $\boldsymbol{B}, \boldsymbol{C}$, Examples of astray ${ }^{\text {te284 }}$ $(\boldsymbol{B})$ and astray $^{\mathrm{tiz} 72 \mathrm{z}}$ (C) larvae treated with zADCY8 morpholino that have weak ipsilateral misprojections. $\boldsymbol{D}$, The percentage of strong ipsilateral misprojections is significantly reduced in both astray mutants compared with wild-type larvae. The numbers of eyes analyzed for each condition are indicated in the bars. The Fisher's exact test was used to determine levels of significance. Shown are dorsal views with anterior to the top. Scale bar, $100 \mu \mathrm{m}$.

\section{Discussion}

Our studies reveal an essential role for calmodulin-activated adenylate cyclases in early retinal pathfinding in the zebrafish embryo. Morpholino knockdown of zADCY8 induces a significant number of retinal ganglion cell axons to be deflected away from the ventral midline instead of following their normal trajectory to the contralateral tectum (Fig. 4). Deflected axons generally join the ipsilateral optic tract and travel to the ipsilateral tectum, but in some instances they travel to entirely inappropriate anterior locations. A second calmodulin-activated cyclase, ADCY1b, acts cooperatively with zADCY8 to facilitate midline crossing of retinal axons (supplemental Fig. S4, available at www.jneurosci.org as supplemental material). The pathfinding errors observed in ADCY8 knockdown embryos arise, at least in part, by a cellautonomous requirement for ADCY8 within retinal ganglion cells. This was demonstrated in three ways. First, ipsilateral misprojections arise from axons of retinal neurons containing zADCY8 morpholino that are transplanted into untreated wildtype embryos (Fig. 5). This indicates that knockdown axons make errors even when interacting with normal host tissues. Second, errors in retinal projections in morpholino-treated embryos were rescued by the reexpression of morpholino-insensitive zADCY8 in retinal neurons (Fig. 6). This demonstrates that zADCY8 expressing axons are more likely to navigate correctly when confronted with knockdown tissues than zADCY8 knockdown axons. Third, experiments in vitro demonstrated that knocking down zADCY8 makes retinal ganglion cells relatively insensitive to SDF1a. This shows that zADCY8 is required within retinal ganglion cells for SDF1 signaling. These findings do not preclude a nonautonomous contribution of ZADCY8 to retinal axon pathfinding in vitro, but they provide decisive support for a retinal ganglion cell-autonomous contribution to normal guidance.

Previous studies have suggested that ADCY1 and ADCY3 play important roles in determining where axons terminate within their targets but have not reported pathfinding errors en route to their targets. Retinal projections have been shown to be perturbed in ADCY1 mutant mice (Welker et al., 1996; Abdel-Majid et al., 1998). Both the orderly retinotopic mapping of retinal projections and the normal separation of ipsilateral and contralateral axons are significantly disrupted in the lateral geniculate of the thalamus (Ravary et al., 2003; Nicol et al., 2006). These alterations in sensory projections have been interpreted as resulting from the presumptive role of ADCY1 in activity-dependent remodeling of sensory connectivity (Ravary et al., 2003; Nicol et al., 2006). Our results suggest the alternative possibility that guidance errors contribute to some of the mapping errors observed in ADCY1 knock-out mice. Abnormal ipsilateral trajectories in the visual system, like those in zADCY8 morphant fish, could lead to the appearance of abnormal segregation of ipsilateral and contralateral projections in the geniculate.

An adenylate cyclase that is indirectly regulated by calmodulin (Wei et al., 1996), ADCY3, has recently been shown to be essential for the normal convergence of olfactory sensory cell axons into specific glomeruli in the main olfactory bulb. ADCY3 is expressed in sensory neurons within the olfactory epithelium (Wong et al., 2000). The axons of many olfactory sensory neurons misproject to multiple abnormal locations in the bulb in ADCY3 ${ }^{-1-}$ mice, and single glomeruli form aberrantly from sensory axons expressing dissimilar olfactory receptors (Chesler et al., 2007; Col et al., 2007; Zou et al., 2007). Guidance of olfactory axon projections in ADCY3 mutant mice may be disrupted by perturbations in cAMP levels that affect how axons respond to guidance cues (Song and Poo, 1999; Chalasani et al., 2003). This could occur if cAMP levels control the expression levels of guidance cue receptors on the surfaces of olfactory axons (Imai et al., 2006). These previously reported roles of ADCY1 and ADCY3 demonstrate their role in determining where axons terminate within their targets. Our results are the first to demonstrate that a calmodulin-activated ADCY is involved in primary axon guidance as axons extend toward their target.

What accounts for the abnormal deflection of retinal axons away from the ventral midline in zADCY8 knockdown embryos? Our findings argue that ADCY8 is required in retinal ganglion cells, and errors arise from either a diminution of retinal sensitivity to midline attractants, the potentiation of retinal responsiveness to midline repellents, or both. Previous work has shown that the chemokine SDF1 makes chick retinal axons less sensitive to multiple repellents in vitro. This effect is mediated by a pertussis toxin- and calmodulin-sensitive elevation of cAMP and the activation of PKA (Chalasani et al., 2003). We hypothesized that calmodulin-activated ADCYs are key components of this antirepellent signaling pathway. If true, then the knockdown of zADCY8 should make zebrafish retinal axons less responsive to SDF1 and more sensitive to slits. Consistent with this prediction, retinal axons harvested from zADCY8 morphants retract in response to a mixture of SDF1 and slit2 much more strongly than retinal axons injected with a control morpholino (Fig. 3). These results show that retinal axons in zADCY8 morphants are insensitive to the antirepellent effects of SDF1 and that this change is cell autonomous.

zADCY8 knockdown makes retinal axons less responsive to SDF1, and consequently more sensitive to slit, in vitro. Can antagonism between SDF1 and slit signaling be observed in vivo? In a previous study, we showed that retinal axons extend ectopically toward an anterior region of slit expression in mutant fish in which slit/robo signaling is reduced. Furthermore, we found that knocking down SDF1/CXCR4 signaling tended to prevent ab- 
normal anterior extension in mutants with reduced robo2, but not in mutants lacking robo2 altogether (Chalasani et al., 2007). Rescue of the anterior misprojection therefore requires the presence of some robo2 activity. These results suggest that SDF1/ CXCR4 signaling antagonizes slit/robo2 signaling and that residual slit/robo2 signaling is boosted by reducing SDF1/CXCR4 signaling. If zADCY8 is a key signaling step in an SDF1 triggered antirepellent pathway, then ZADCY8 knockdown should also tend to rescue anterior misprojections in mutants that have a partial, but not a total, loss of robo2. This prediction was confirmed by experiment in this study. zADCY8 knockdown rescues anterior retinal misprojections in partial but not complete loss of robo2 function mutants (Fig. 7). Both our in vitro and our in vivo findings therefore support a model in which zADCY8 constitutes a key intermediate in an SDF1-activated antirepellent signaling pathway.

The generalization of this same argument as applied to other potential antagonists of slit signaling at the midline could explain why zADCY8 knockdown induces midline deflection of retinal axons. Slits expressed near the midline play an important role in determining where the optic chiasm forms (Erskine et al., 2000; Niclou et al., 2000; Hutson and Chien, 2002; Plump et al., 2002; Chalasani et al., 2007). Disruptions of slit1 and slit2 in mice induce retinal defasciculation and ectopic crossings at the chiasm (Plump et al., 2002), whereas loss of the slit receptor robo2 induces widening of the chiasm and ectopic projections of retinal axons to inappropriate targets (Plachez et al., 2008). Retinal axons cross the ventral midline in unusual locations and project ectopically in zebrafish mutants with reduced or missing robo2 (Fricke et al., 2001; Hutson and Chien, 2002). The chiasm normally forms where slit expression is low (Erskine et al., 2000; Niclou et al., 2000; Hutson and Chien, 2002; Plump et al., 2002), but since slits are secreted and are expressed in nearby midline tissues, it is likely that low levels of slit proteins are present even at the chiasm where retinal axons cross. One plausible explanation for ipsilateral misrouting of retinal projections in zADCY8 morphants is that a loss of antirepellent signaling makes retinal axons hypersensitive to slits in the chiasm.

If zADCY8 knockdown induces abnormal ipsilateral projections by making retinal axons overly sensitive to midline slits, then a reduction in slit/robo signaling would be predicted to rescue these errors. Supporting this idea is the finding that zADCY8 morpholino-induced ipsilateral projections are much less frequent in mutant embryos in which robo 2 is either reduced or absent. Although our findings are consistent with the idea that ADCY8 knockdown makes retinal axons hypersensitive to midline repellents, we cannot exclude the alternative possibility that loss of zADCY8 interferes with an attractive mechanism that helps retinal axons cross the midline. Whether ZADCY8 is required for an antirepellent or an attractive response to a midline cue, that cue is unlikely to be SDF1. SDF1 is not expressed at the midline and ipsilateral misprojections are not observed in either SDF1 or cxcr4b mutants or morphants (Li et al., 2005). Several G-protein-coupled signaling pathways have been proposed to affect retinal axon guidance. The adenosine receptor $A 2 b$ is thought to have a positive influence on cAMP levels in retinal axons that modulates their response to netrin (Shewan et al., 2002), whereas sonic hedgehog is thought to mediate a repellent response by reducing cAMP levels in retinal growth cones (Trousse et al., 2001). Activation of the metabotropic glutamate receptor $\mathrm{mGluR} 1$ reduces the repellent activity of slit 2 on retinal axons in a cAMP-dependent manner (Kreibich et al., 2004). It is not known whether any of these signals influence ADCY1 or
ADCY8 activity, nor have any of these GPCRs been reported to affect axonal crossing at the midline.

Studies in vitro have demonstrated that modulating cAMP levels within the growth cone can have a profound effect on axon guidance, but the role of cAMP in axonal pathfinding in vivo has been less well studied. Our findings demonstrate an essential and previously unappreciated role for calmodulin-activated adenylate cyclases ADCY1b and ADCY8 in retinal axon pathfinding in vivo. Furthermore, they show that ADCY8 is part of a signaling pathway that antagonizes repellent signaling. They support the idea that retinal axon sensitivity to midline guidance cues is regulated by cAMP levels. Future studies will focus on the identification of additional cues, in addition to the chemokine SDF1, that influence axonal pathfinding through the activation of ADCY8.

\section{References}

Abdel-Majid RM, Leong WL, Schalkwyk LC, Smallman DS, Wong ST, Storm DR, Fine A, Dobson MJ, Guernsey DL, Neumann PE (1998) Loss of adenylyl cyclase I activity disrupts patterning of mouse somatosensory cortex. Nat Genet 19:289-291.

Burrill JD, Easter SS Jr (1995) The first retinal axons and their microenvironment in zebrafish: cryptic pioneers and the pretract. J Neurosci 15:2935-2947.

Chalasani SH, Sabelko KA, Sunshine MJ, Littman DR, Raper JA (2003) A chemokine, SDF-1, reduces the effectiveness of multiple axonal repellents and is required for normal axon pathfinding. J Neurosci 23:1360-1371.

Chalasani SH, Sabol A, Xu H, Gyda MA, Rasband K, Granato M, Chien CB, Raper JA (2007) Stromal cell-derived factor-1 antagonizes slit/robo signaling in vivo. J Neurosci 27:973-980.

Chesler AT, Zou DJ, Le Pichon CE, Peterlin ZA, Matthews GA, Pei X, Miller MC, Firestein S (2007) A G protein/cAMP signal cascade is required for axonal convergence into olfactory glomeruli. Proc Natl Acad Sci U S A 104:1039-1044.

Col JA, Matsuo T, Storm DR, Rodriguez I (2007) Adenylyl cyclasedependent axonal targeting in the olfactory system. Development 134:2481-2489.

David NB, Sapède D, Saint-Etienne L, Thisse C, Thisse B, Dambly-Chaudière C, Rosa FM, Ghysen A (2002) Molecular basis of cell migration in the fish lateral line: role of the chemokine receptor CXCR4 and of its ligand, SDF1. Proc Natl Acad Sci U S A 99:16297-16302.

Dickson BJ (2002) Molecular mechanisms of axon guidance. Science 298:1959-1964.

DiPilato LM, Zhang J (2009) The role of membrane microdomains in shaping beta2-adrenergic receptor-mediated cAMP dynamics. Mol Biosyst 5:832-837.

Dwinell MB, Ogawa H, Barrett KE, Kagnoff MF (2004) SDF-1/CXCL12 regulates cAMP production and ion transport in intestinal epithelial cells via CXCR4. Am J Physiol Gastrointest Liver Physiol 286:G844-G850.

Erskine L, Williams SE, Brose K, Kidd T, Rachel RA, Goodman CS, TessierLavigne M, Mason CA (2000) Retinal ganglion cell axon guidance in the mouse optic chiasm: expression and function of robos and slits. J Neurosci 20:4975-4982.

Ferguson GD, Storm DR (2004) Why calcium-stimulated adenylyl cyclases? Physiology (Bethesda) 19:271-276

Fricke C, Lee JS, Geiger-Rudolph S, Bonhoeffer F, Chien CB (2001) astray, a zebrafish roundabout homolog required for retinal axon guidance. Science 292:507-510.

Gu C, Cooper DM (1999) Calmodulin-binding sites on adenylyl cyclase type VIII. J Biol Chem 274:8012-8021.

Hendricks M, Jesuthasan S (2007) Electroporation-based methods for in vivo, whole mount and primary culture analysis of zebrafish brain development. Neural Dev 2:6.

Hutson LD, Chien CB (2002) Pathfinding and error correction by retinal axons: the role of astray/robo2. Neuron 33:205-217.

Hutson LD, Jurynec MJ, Yeo SY, Okamoto H, Chien CB (2003) Two divergent slit1 genes in zebrafish. Dev Dyn 228:358-369.

Imai T, Suzuki M, Sakano H (2006) Odorant receptor-derived cAMP signals direct axonal targeting. Science 314:657-661. 
Kimmel CB, Ballard WW, Kimmel SR, Ullmann B, Schilling TF (1995) Stages of embryonic development of the zebrafish. Dev Dyn 203:253-310.

Kozlowski DJ, Murakami T, Ho RK, Weinberg ES (1997) Regional cell movement and tissue patterning in the zebrafish embryo revealed by fate mapping with caged fluorescein. Biochem Cell Biol 75:551-562.

Kreibich TA, Chalasani SH, Raper JA (2004) The neurotransmitter glutamate reduces axonal responsiveness to multiple repellents through the activation of metabotropic glutamate receptor 1. J Neurosci 24:7085-7095.

Lee JS, Ray R, Chien CB (2001) Cloning and expression of three zebrafish roundabout homologs suggest roles in axon guidance and cell migration. Dev Dyn 221:216-230.

Li Q, Shirabe K, Thisse C, Thisse B, Okamoto H, Masai I, Kuwada JY (2005) Chemokine signaling guides axons within the retina in zebrafish. J Neurosci 25:1711-1717.

Liu Y, Berndt J, Su F, Tawarayama H, Shoji W, Kuwada JY, Halloran MC (2004) Semaphorin3D guides retinal axons along the dorsoventral axis of the tectum. J Neurosci 24:310-318.

Marcus RC, Shimamura K, Sretavan D, Lai E, Rubenstein JL, Mason CA (1999) Domains of regulatory gene expression and the developing optic chiasm: correspondence with retinal axon paths and candidate signaling cells. J Comp Neurol 403:346-358.

Morcos PA (2007) Achieving targeted and quantifiable alteration of mRNA splicing with Morpholino oligos. Biochem Biophys Res Commun 358: 521-527.

Mullins MC, Hammerschmidt M, Haffter P, Nüsslein-Volhard C (1994) Large-scale mutagenesis in the zebrafish: in search of genes controlling development in a vertebrate. Curr Biol 4:189-202.

Niclou SP, Jia L, Raper JA (2000) Slit2 is a repellent for retinal ganglion cell axons. J Neurosci 20:4962-4974.

Nicol X, Bennis M, Ishikawa Y, Chan GC, Repérant J, Storm DR, Gaspar P (2006) Role of the calcium modulated cyclases in the development of the retinal projections. Eur J Neurosci 24:3401-3414.

Nielsen MD, Chan GC, Poser SW, Storm DR (1996) Differential regulation of type I and type VIII Ca ${ }^{2+}$-stimulated adenylyl cyclases by Gi-coupled receptors in vivo. J Biol Chem 271:33308-33316.

Park HC, Kim CH, Bae YK, Yeo SY, Kim SH, Hong SK, Shin J, Yoo KW, Hibi M, Hirano T, Miki N, Chitnis AB, Huh TL (2000) Analysis of upstream elements in the $\mathrm{HuC}$ promoter leads to the establishment of transgenic zebrafish with fluorescent neurons. Dev Biol 227:279-293.

Piper M, van Horck F, Holt C (2007) The role of cyclic nucleotides in axon guidance. Adv Exp Med Biol 621:134-143.

Pittman AJ, Law MY, Chien CB (2008) Pathfinding in a large vertebrate axon tract: isotypic interactions guide retinotectal axons at multiple choice points. Development 135:2865-2871.

Plachez C, Andrews W, Liapi A, Knoell B, Drescher U, Mankoo B, Zhe L, Mambetisaeva E, Annan A, Bannister L, Parnavelas JG, Richards LJ, Sundaresan V (2008) Robos are required for the correct targeting of retinal ganglion cell axons in the visual pathway of the brain. Mol Cell Neurosci 37:719-730.

Plump AS, Erskine L, Sabatier C, Brose K, Epstein CJ, Goodman CS, Mason CA, Tessier-Lavigne M (2002) Slit1 and Slit2 cooperate to prevent premature midline crossing of retinal axons in the mouse visual system. Neuron 33:219-232.
Ravary A, Muzerelle A, Hervé D, Pascoli V, Ba-Charvet KN, Girault JA, Welker E, Gaspar P (2003) Adenylate cyclase 1 as a key actor in the refinement of retinal projection maps. J Neurosci 23:2228-2238.

Rhoads AR, Friedberg F (1997) Sequence motifs for calmodulin recognition. FASEB J 11:331-340.

Robu ME, Larson JD, Nasevicius A, Beiraghi S, Brenner C, Farber SA, Ekker SC (2007) p53 activation by knockdown technologies. PLoS Genet 3:e78.

Sakai JA, Halloran MC (2006) Semaphorin 3d guides laterality of retinal ganglion cell projections in zebrafish. Development 133:1035-1044.

Shewan D, Dwivedy A, Anderson R, Holt CE (2002) Age-related changes underlie switch in netrin-1 responsiveness as growth cones advance along visual pathway. Nat Neurosci 5:955-962.

Simpson RE, Ciruela A, Cooper DM (2006) The role of calmodulin recruitment in $\mathrm{Ca}^{2+}$ stimulation of adenylyl cyclase type 8 . J Biol Chem 281:17379-17389.

Song HJ, Poo MM (1999) Signal transduction underlying growth cone guidance by diffusible factors. Curr Opin Neurobiol 9:355-363.

Tessier-Lavigne M, Goodman CS (1996) The molecular biology of axon guidance. Science 274:1123-1133.

Trousse F, Martí E, Gruss P, Torres M, Bovolenta P (2001) Control of retinal ganglion cell axon growth: a new role for Sonic hedgehog. Development 128:3927-3936.

Vorherr T, Knöpfel L, Hofmann F, Mollner S, Pfeuffer T, Carafoli E (1993) The calmodulin binding domain of nitric oxide synthase and adenylyl cyclase. Biochemistry 32:6081-6088.

Warrington NM, Woerner BM, Daginakatte GC, Dasgupta B, Perry A, Gutmann DH, Rubin JB (2007) Spatiotemporal differences in CXCL12 expression and cyclic AMP underlie the unique pattern of optic glioma growth in neurofibromatosis type 1. Cancer Res 67:8588-8595.

Wayman GA, Impey S, Wu Z, Kindsvogel W, Prichard L, Storm DR (1994) Synergistic activation of the type I adenylyl cyclase by $\mathrm{Ca}^{2+}$ and Gscoupled receptors in vivo. J Biol Chem 269:25400-25405.

Wei J, Wayman G, Storm DR (1996) Phosphorylation and inhibition of type III adenylyl cyclase by calmodulin-dependent protein kinase II in vivo. J Biol Chem 271:24231-24235.

Welker E, Armstrong-James M, Bronchti G, Ourednik W, GheorghitaBaechler F, Dubois R, Guernsey DL, Van der Loos H, Neumann PE (1996) Altered sensory processing in the somatosensory cortex of the mouse mutant barrelless. Science 271:1864-1867.

Willoughby D, Cooper DM (2007) Organization and $\mathrm{Ca}^{2+}$ regulation of adenylyl cyclases in cAMP microdomains. Physiol Rev 87:965-1010.

Wong ST, Trinh K, Hacker B, Chan GC, Lowe G, Gaggar A, Xia Z, Gold GH, Storm DR (2000) Disruption of the type III adenylyl cyclase gene leads to peripheral and behavioral anosmia in transgenic mice. Neuron 27:487-497.

Xiao T, Roeser T, Staub W, Baier H (2005) A GFP-based genetic screen reveals mutations that disrupt the architecture of the zebrafish retinotectal projection. Development 132:2955-2967.

Zou DJ, Chesler AT, Le Pichon CE, Kuznetsov A, Pei X, Hwang EL, Firestein $S$ (2007) Absence of adenylyl cyclase 3 perturbs peripheral olfactory projections in mice. J Neurosci 27:6675-6683. 